\title{
Large-scale ensemble averaging of ambulatory impedance cardiograms
}

\author{
HARRIËTTE RIESE, PAUL F. C. GROOT, MIREILLE vAN DEN BERG, NINA H. M. KUPPER, \\ ELLIS H. B. MAGNEE, and ELLEN J. ROHAAN \\ Vrije Universiteit, Amsterdam, The Netherlands \\ TANJA G. M. VRIJKOTTE \\ Academisch Medisch Centrum, Amsterdam, The Netherlands \\ and \\ GONNEKE WILLEMSEN and ECO J. C. DE GEUS \\ Vrije Universiteit, Amsterdam, The Netherlands
}

\begin{abstract}
Impedance cardiography has been used increasingly to measure human physiological responses to emotional and mentally engaging stimuli. The validity of large-scale ensemble averaging of ambulatory impedance cardiograms was evaluated for preejection period (PEP), interbeat interval, and dZ/dt ${ }_{(\min )}$ amplitude. We tested whether the average of "classical" 60 -sec ensemble averages across periods with fixed activity, posture, physical load, social situation, and location could be accurately estimated from a single large-scale ensemble average spanning these entire periods. Impedance and electrocardiograms were recorded for about 24 -h from 21 subjects. Recordings were scored by seven raters, using both methods for each subject. Good agreement (average intraclass correlation coefficient was .91) between both ensemble averaging methods was found for all three cardiac function measures. The results indicate that for unambiguous ambulatory impedance cardiograms, large-scale ensemble averaging is valid, which makes measuring prolonged changes in cardiac sympathetic activity by measuring ambulatory PEP feasible even in large epidemiological samples.
\end{abstract}

Impedance cardiography has been used increasingly to measure human physiological responses to emotional and mentally engaging stimuli (e.g., Alfie et al., 1995; Bosch et al., 2001; Bosch et al., 2000; Burgess, Kleiman, \& Trinder, 1999; Burgess, Sletten, Savic, Gilbert, \& Dawson, 2001; Burgess, Trinder, \& Kim, 1999; Burgess, Trinder, Kim, \& Luke, 1997; Groza, Vrancianu, Lazar, Baevski, \& Funtova, 1984; A. S. Harris, Burgess, \& Dawson, 2001; Holmes, Burgess, \& Dawson, 2002; Houtveen, Rietveld, $\&$ de Geus, 2002; Kizakevich et al., 2000; Light, Kothandapani, \& Allen, 1998; Matthews, Flory, Owens, Harris, \& Berga, 2001; Neumann \& Waldstein, 2001; Ring et al., 1999; Sherwood, Allen, Obrist, \& Langer, 1986). The impedance cardiogram (ICG) is defined as the first derivative of pulsatile changes in the transthoracic impedance $(\mathrm{dZ} / \mathrm{dt})$. From the ICG, the systolic time intervals preejection period (PEP) and left ventricular ejection time can be derived, as well as $\mathrm{dZ} / \mathrm{dt}_{(\min )}$ amplitude and stroke volume. In this paper we focus specifically on the PEP. The PEP is an index of cardiac contractility (Siegel \& Sonnenblick, 1963) introduced to human psychophysiology by Newlin and Levenson in 1979. In 1990, solid

Correspondence should be addressed to H. Riese, Department of Biological Psychology, Vrije Universiteit, Van der Boechorststraat 1, 1081 BT Amsterdam, The Netherlands (e-mail: h.riese@psy.vu.nl). guidelines for impedance cardiography and signal analyses were published in Psychophysiology (Sherwood et al., 1990), and many further technical and methodological refinements have been implemented since then (Kelsey \& Guethlein, 1990; Kelsey et al., 1998).

So far, virtually all studies have assessed PEP reactivity in the laboratory using brief stressors and brief pretask and posttask rest periods (e.g., Bosch et al., 2000; Light et al., 1998; Matthews et al., 2001; Neumann \& Waldstein, 2001; Ring et al., 1999). This leaves uncharted how PEP reacts in response to much longer exposure to stress, such as that which occurs in the course of a stressful work day, or whether PEP shows evidence of recovery from daytime sympathetic activation or other compensatory reactions during the night. The study of these more chronic changes requires ambulatory monitoring, which has greater ecological validity, increases reliability via the collection of multiple values, and allows analyses of the effects of psychological processes over a longer time scale (Staessen et al., 2001; Verdecchia, 2000; Vrijkotte, van Doornen, \& de Geus, 2000).

Recently, various systems have become available that would allow the ambulatory monitoring of thoracic ICGs (Cybulski, 2000; Nakonezny et al., 2001; Sherwood, McFetridge, \& Hutcheson, 1998). We have developed an ambulatory monitoring device (VU-AMS; Vrije Universiteit-Ambulatory Monitoring System, Version 4.6, VU- 
FPP, Amsterdam) for combined electrocardiogram(EKG) and ICG recording from six spot electrodes (Willemsen, de Geus, Klaver, van Doornen, \& Carroll, 1996). A number of small-scale studies have demonstrated the feasibility of this device in ambulatory settings and have shown that changes in PEP due to mental load, posture, and exercise can be picked up reliably (de Geus \& van Doornen, 1996; Willemsen et al., 1996). Yet, ambulatory 24-h PEP recording has its largest potential value in large-scale epidemiological settings, where it can be made to accompany the "routine" ambulatory blood pressure and heart rate recording already performed on this scale. An example is our ongoing Netherlands twin family study of anxious depression (NETSAD), in which ambulatory blood pressure and ambulatory PEP are being collected in nearly 1,000 twins and siblings selected on the basis of high or low genetic susceptibility for depression (Boomsma et al., 2000).

The usual approach to ICG data reduction-ensemble averaging over 60 -sec periods-is implemented in the VU-AMS at the hardware level. Ensemble averaging involves digitizing the EKG and the ICG over all beats in a 60 -sec period with respect to the peak of the EKG R-wave, and then averaging by summing the digitized samples for each signal and dividing by the number of synchronized beats. This procedure reduces the impact of single-beat fluctuations in the impedance signal through respiration and slow thorax movement. Systolic time intervals scored in the resulting 60-sec ensemble-averaged ICG correspond very closely to the mean systolic time intervals obtained over the (reliable) single-beat ICGs in that same minute (Boomsma, de Vries, \& Orlebeke, 1989; Kelsey \& Guethlein, 1990, Kelsey et al., 1998; Muzi et al., 1985). The number of 60-sec ensemble-averaged ICG waveforms for a single subject for a single measurement collected during a typical 24-h ambulatory recording day can be up to 1,440 . A mean of 1,263 ICG waveforms is available for 750 subjects from the NETSAD sample; thus a total of 947,250 waveforms are already available, from which a 60-sec ensemble-averaged PEP can be extracted. Although the scoring of the impedance waveform has been automated by a number of algorithms, the consensus is that PEP scoring from the ICG waveform needs to be inspected visually. The labor intensiveness of manually scoring 24-h ambulatory PEP in large samplesfor instance, in our NETSAD subjects-renders 60-sec ensemble averaging of the ambulatory ICG virtually impossible.

The large-scale ensemble averaging strategy exploits the idea that most ambulatory studies will ultimately average the results obtained on the smaller time scale (e.g., 60-sec averages) over much longer time periods. Usually consecutive fragments of ambulatory recording are identified in which no significant change occurs in the hypothesized causes of intraindividual variance in ICG-derived variables-for instance, posture, type of activity, physical load, social situation, location, or level of self-experienced mental or emotional strain. Such fragments, which are usually between $5 \mathrm{~min}$ and an hour in length, will be referred to as fixed periods henceforth. Most studies using the PEP to understand the role of the sympathetic nervous system in health and disease tend to use the average of the 60-sec ensemble averages for PEP across these fixed periods as their final unit of statistical analysis. This applies to between(e.g., high- vs. low-neurotic subjects) as well as withinsubjects (e.g., workday vs. nonworkday) designs.

Using the data from our NETSAD sample, we aimed to test whether the mean PEP of the 60-sec ensemble averages across a fixed period could be accurately estimated from a single large-scale ensemble average spanning the entire fixed period. This approach takes the same ensemble averaging strategy used to obtain 60-sec averages from single-beat waveforms and applies it to the 60-sec ensemble-averaged waveforms to obtain a larger scale ensemble average of up to $1 \mathrm{~h}$. Large-scale ensemble averaging, if it validly recaptures the information in the original 60-sec ensemble averages, would substantially reduce the total amount of visual inspection needed and hence make ambulatory ICG feasible for epidemiology scaled studies without the necessity of depending entirely on prohibitively laborious interactive scoring.

\section{METHOD}

\section{Subjects}

For the present study, 21 subjects ( 7 men and 14 women) were randomly selected from a larger sample (NETSAD) participating in a study on the genetics of anxious depression and cardiovascular disease. The age range was 20 to 40 years, with a mean of $29(S D=$ 5.14). The BMI range was 18.59 to 27.47 , with a mean of 22.14 $(S D=2.72)$. The ethics committee of the Vrije Universiteit approved the protocol for this study and all subjects gave written consent.

\section{Procedure}

Subjects were visited at home between 7 and 10 a.m. and fitted with the VU-AMS device. They received detailed instructions to regularly check the "all clear" signal of the device (a small blinking light on the side of the device) and how to proceed in case of device malfunction. Changes in physical activity and posture are important determinants of changes in the EKG and ICG signals. Since activity cannot be controlled or kept constant in an ambulatory setting, subjects were instructed to carefully keep an activity diary. Every 30 ( \pm 10 randomized) min (prompted by a beep from the VUAMS device), subjects had to write down the time, location, activities, bodily postures, and social situation they had been engaged during the previous half hour, in chronological order.

\section{Ambulatory Monitoring}

The EKG and ICG signals were recorded continuously by the VU-AMS from a six-spot electrode configuration. For the EKG, two active electrodes and one common electrode were positioned to maximize the $\mathrm{R}$-wave amplitude. One active electrode was placed at the jugular notch of the sternum between the collar bones and the other one below the left breast, $4 \mathrm{~cm}$ beneath the nipple. The ground electrode was placed equidistant between the active electrodes at the right side of the chest, between the lower two ribs. The EKG signal was led into a differential amplifier, with an input impedance higher than $1 \mathrm{M} \Omega$, and a CMRR of $70 \mathrm{~dB}$. The amplified EKG is passed through a second-order bandpass filter of $17 \mathrm{~Hz}$, and the filtered signal is used for R-peak triggering. The R-peak is recognized with a level detector with automatic level adjustment. The output of 
the level detector is connected to an interrupt request (IRQ) line of the microprocessor. At each R-peak, a millisecond counter is read and reset, yielding the interbeat interval (IBI) time series at 1-msec resolution. Only this IBI time series is stored, not the full EKG. Reliability and validity aspects of heart rate (variability) recording with the VU-AMS have been described previously (de Geus, Willemsen, Klaver, \& van Doornen, 1995; Vrijkotte, Riese, \& de Geus, 2001).

Thoracic impedance $(\mathrm{Z})$ was assessed against a constant current of $50 \mathrm{KHz}, 350$ microamperes. Two electrodes on the back were used for sending this high-frequency current through the subject's body, with two measuring electrodes on the chest picking up the voltage drop over the thorax. The upper current electrode on the back was placed at least $3 \mathrm{~cm}$ above the horizontal plane of the upper measuring electrode. The lower current electrode on the back was placed at least $3 \mathrm{~cm}$ below the horizontal plane of the lower measuring electrode. The upper measuring electrode and the ICG lead wire were used for ICG and EKG pickup. The lower measuring electrode was placed at the tip of the sternum (xiphoid process). The highfrequency impedance signal was amplified and led to a precision rectifier. The rectified signal was filtered at $72 \mathrm{~Hz}$ (low pass) to give basal thoracic impedance $\mathrm{Z}$. Filtering $\mathrm{Z}$ at $0.1 \mathrm{~Hz}$ (high pass) supplies $\Delta \mathrm{Z}$, which in return was filtered at $30.0 \mathrm{~Hz}$ (high pass) to determine $\mathrm{dZ} / \mathrm{dt}$. A $\mathrm{Z} 0$ value was determined by averaging $\mathrm{Z}$ samples at the R-wave, $\Delta \mathrm{Z}$ and $\mathrm{dZ} / \mathrm{dt}$ were led to an $\mathrm{A} / \mathrm{D}$ converter of the microprocessor and digitized at 10 and $250 \mathrm{~Hz}$, respectively. The value of the $\mathrm{dZ} / \mathrm{dt}_{(\min )}$ was calculated as the amplitude span between the $\mathrm{dZ} / \mathrm{dt}$ peak and the $\mathrm{dZ} / \mathrm{dt}=0$ point. An average ICG waveform of 128 samples $(512 \mathrm{msec})$ was calculated by adding all individual R-wavelocked dZ/dt waveforms during each 1-min period and dividing by the total number of beats in that minute. This ensembled $\mathrm{dZ} / \mathrm{dt}$ waveform will be referred to as a "60-sec ensemble average."

To index gross body movements (i.e., transitions in posture and activity), the VU-AMS device contains an accelerometer sensitive to changes in vertical acceleration. Its output is amplified, rectified, and led into a hardware integrator. Every $5 \mathrm{sec}$ this integrator is sampled and reset by the microprocessor. The integrated values are averaged up to the user-defined sample interval and have a range of 0 to $3.2 \mathrm{gsec}$ with a resolution of $0.008 \mathrm{gsec}$. Average body movement over 30 -sec periods was stored throughout the 24 -h recording time and used to verify the changes in postures and activities reported in the activity diaries.

\section{ICG Waveform Scoring}

An interactive program was used to graphically display the 128 $\mathrm{dZ} / \mathrm{dt}$ samples that make up the ICG waveforms. Figure 1A plots a typical 60-sec ensemble average. The graph also shows three vertical lines, representing (1) upstroke or B-point, (2) dZ/dt $\mathrm{dmin}_{\text {) }}$, and (3) incisura, or X-point. Two cardiac function measures were obtained from these points: (1) the PEP (in milliseconds) and (2) the peak of the $\mathrm{dZ} / \mathrm{dt}$ waveform, $\mathrm{dZ} / \mathrm{dt}_{(\min )}$ (in $\Omega / \mathrm{sec}$ ). The PEP is defined as the period between the onset of the electromechanical systole (EMS) and the onset of left ventricular ejection at the opening of the aortic valves. Impedance cardiography usually estimates PEP as the time interval between the EKG Q-wave (onset EMS) and the B-point in the ICG, which is the start of the rapid upslope of $\mathrm{dZ} / \mathrm{dt}$ to its maximum value (Sherwood et al., 1990). However, in the normal physiological range, there is little variation during the $\mathrm{Q}$-onset to $\mathrm{R}$-wave time interval within or between subjects (Willemsen et al., 1996). Sherwood et al. (1990) have already suggested the use of the abbreviated PEP (R-wave to $\mathrm{dZ} / \mathrm{dt}$ point) to index myocardial contractility because the R-wave is clearly defined in all subjects, whereas Q-onset may be unreliable in some. The VU-AMS, therefore, does not record the full EKG but instead uses the R-wave as an approximation of the onset of the EMS and PEP scoring in the VUAMS relative to the R-wave. We add a fixed Q- $\mathrm{R}$ interval of $48 \mathrm{msec}$ to this abbreviated PEP to allow easy comparison with the usual Q-wave-based PEP.

Reliability of B-point detection is limited by the fact that the Bpoint is not always well defined (Debski, Zhang, Jennings, \& Kamarck, 1993). Ambiguity in the location of the B-point presents a serious problem in impedance scoring and has prompted us (and others; e.g., Kelsey \& Guethlein, 1990, Kelsey et al., 1998) to adopt a procedure of fully interactive visual inspection to check and correct automated scoring. First, ensemble averages that do not resemble the typical morphology of a $\mathrm{dZ} / \mathrm{dt}$ waveform are deleted. Next, a new B-point is set manually for ensemble averages where automated scoring is seen to choose B-points that are not morphologically consistent across the entire recording.

\section{Large-Scale Ensemble Averaging}

Using the activity diary entries in combination with a visual display of the vertical accelerometer signal, which is sensitive to gross body movements and the heart rate, the entire recording was divided into fixed periods (i.e., periods with fixed activity, posture, physical load, location, and social situation). Table 1 shows examples of the various categories used for coding these fixed periods. If fixed periods lasted more than $1 \mathrm{~h}$ (e.g., during sleep), they were divided into multiple periods of maximally $1 \mathrm{~h}$. Table 2 provides an example of the coding of fixed periods during a 24-h registration. The upper part of Figure 2 depicts how various 60 -sec ensemble averages in a part of an ambulatory recording are grouped into two fixed periods. Usually, statistical analyses are based on the mean value of all 60-sec ensemble averages across such a fixed period. The lower part of Figure 2 presents our alternative approach, where data reduction is performed by large-scale ensemble averaging all 60 -sec ensemble-averaged waveforms across a period with fixed activity, posture, physical load, location, and social situation.

In the approach just described, each of the 60 -sec ensemble averages is part of a larger period of fixed activity, posture, location, and social situation. Figure 1B shows a 60 -sec ensemble average and the large-scale ensemble average to which this 60 -sec period contributed. In general, the morphology of large-scale ensemble averages strongly resembles that of the 60 -sec ensemble averages, with amplitudes of peaks and troughs slightly attenuated. Both automated and visual scoring was done according to the rules applied to the 60 -sec ensemble averages.

\section{Multiple Raters Design}

Seven ("A"-"G") experienced VU-AMS users interactively inspected and corrected the automatically scored $\mathrm{dZ} / \mathrm{dt}$ waveforms of the 21 subjects. Two raters were randomly assigned to each subject. For each subject, two raters inspected and corrected both the 60-sec ensemble averages and the large-scale ensemble averages, in independent runs (i.e., without using the combined traces shown in Figure 1B). Thus the scored ICG signal of each subject was inspected and corrected four times (two raters using two different ensemble averaging methods).

\section{Statistical Analysis}

Validity of the large-scale ensemble averaging method for IBI, PEP, and $\mathrm{dZ} / \mathrm{dt}$ amplitude was determined separately for each subject by computing the intraclass correlation coefficients between the means of the 60-sec and large-scale ensemble averages across all fixed periods. In addition, interrater reliability was determined for both ensemble averaging methods. The intraclass correlations were computed between all 60 -sec ensemble averages of Raters 1 and 2 and of all large-scale ensemble averages of Raters 1 and 2, again separately for each subject.

To test for a possible effect of activity/posture on the comparability of the ensembling methods, a three-level repeated measures multivariate analysis of variance (MANOVA) with method of scoring 
A

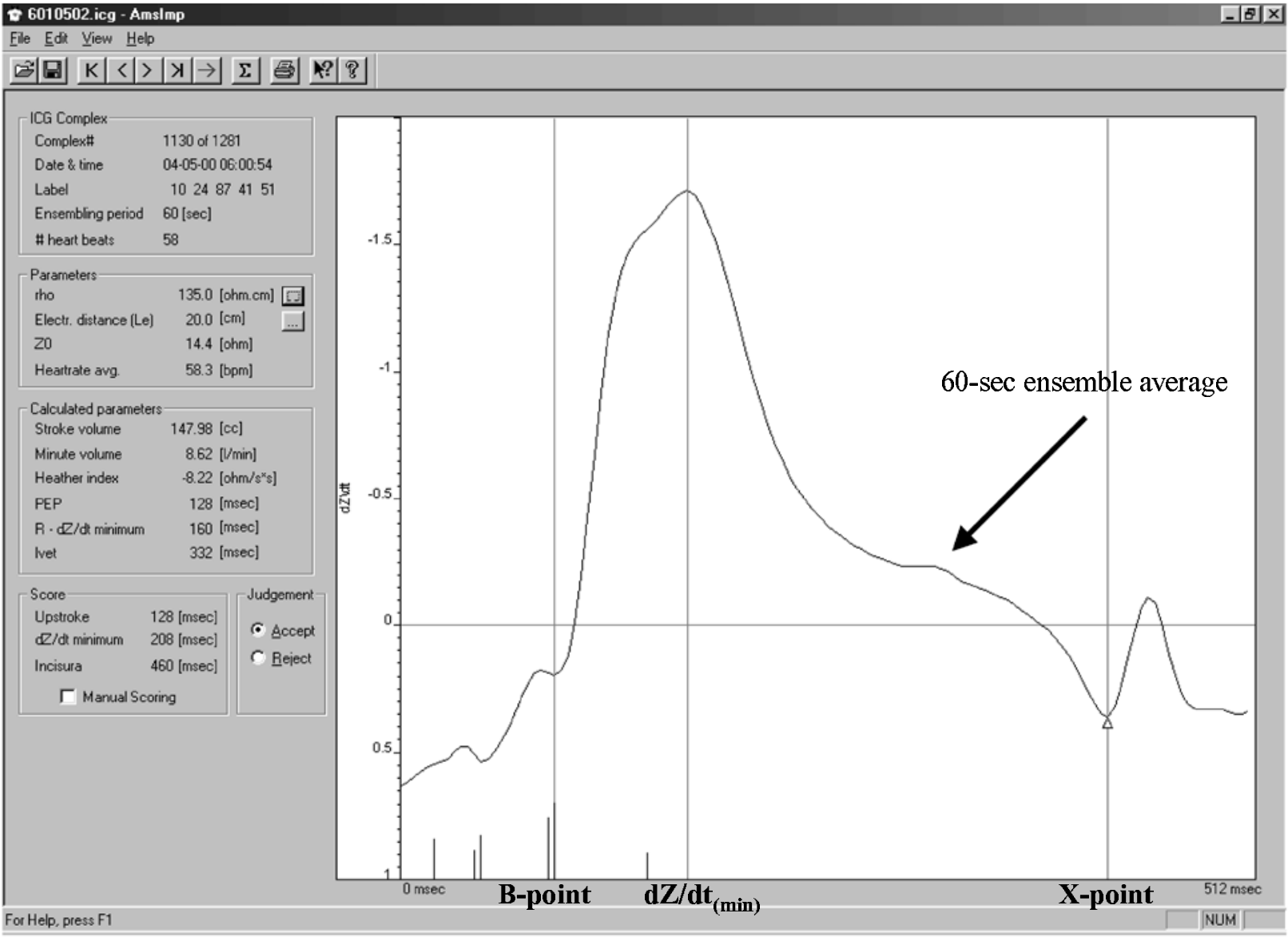

Figure 1A. Graph of a 60 -sec ensemble average obtained by ambulatory impedance cardiography. The three vertical bars indicate the B-point, $\mathrm{dZ} / \mathrm{dt}_{(\mathrm{min})}$, and $\mathrm{X}$-point scored on the large-scale ensemble average. Note: The black spikes at the bottom of the graph represent candidate $B$-points calculated by an automatic scoring algorithm; the program automatically sets the most likely of these points to be the upstroke. The candidates were derived as the zero crossings in the first (throughout, peaks are discarded) and second derivative (inflection) of the $\mathrm{dZ} / \mathrm{dt}$ waveform in the interval between the $\mathrm{R}$-wave and the $\mathrm{dZ} / \mathrm{dt}_{(\mathrm{min})}$. The height of these spikes indicates the likelihood that a candidate is the actual B-point. The algorithm setting this likelihood takes various factors into account, including the length of the uphill slope following the candidate, the closeness to the $\mathrm{dZ} / \mathrm{dt}$ zero line, the closeness to the location of the $\mathrm{B}$ point in the surrounding 60 -sec ensembles, and the length of the total interbeat interval (IBI).

(60-sec vs. large-scale ensemble average), rater (Rater 1 vs. Rater 2), and posture/physical load (lying, sitting, or upright activities) was used. The latter analysis combined the data from all 21 subjects. All analyses were performed in SPSS Version 11.0 for Windows.

\section{RESULTS}

The number of 60-sec ensemble averages varied across subjects from 1,153 to 1,539 , with a mean of $1,364.4(S D=$ 93.8). Of all 60 -sec ensemble averages, $35.4 \%$ were obtained during sleep, $26.9 \%$ were obtained during sitting, $17.6 \%$ during standing or walking, and the remaining $20.1 \%$ during activities with a varying degree of physical load (e.g., $1.1 \%$ bicycling). During interactive visual inspection, $4.8 \%$ of these 60 -sec ensemble averages were rejected. The number of large-scale ensemble averages varied between 27 and 60, with a mean of $36.2(S D=$
7.3 ) per subject; $1.7 \%$ of the large-scale ensemble averages were rejected during interactive visual inspection.

\section{Interrater Agreement}

The mean intraclass correlation coefficients between cardiac measures scored by two different raters were .75 for PEP, .99 for IBI, and .99 for $\mathrm{dZ} / \mathrm{dt}$ for the 60 -sec ensemble averaging method. For the large-scale ensemble averaging method, mean intraclass correlation coefficients were .68 (PEP), .99 (IBI), and .99 (dZ/dt). Of these coefficients, $80.2 \%$ were higher than .80 , and $78.6 \%$ higher than .90 , indicating that the relative ranking of the cardiac function measures remained fairly constant regardless of the researcher who scored the data. Low correlation coefficients for PEP were found, however, for Subjects 5, 10, and 12 for the 60-sec ensemble averaging 
B

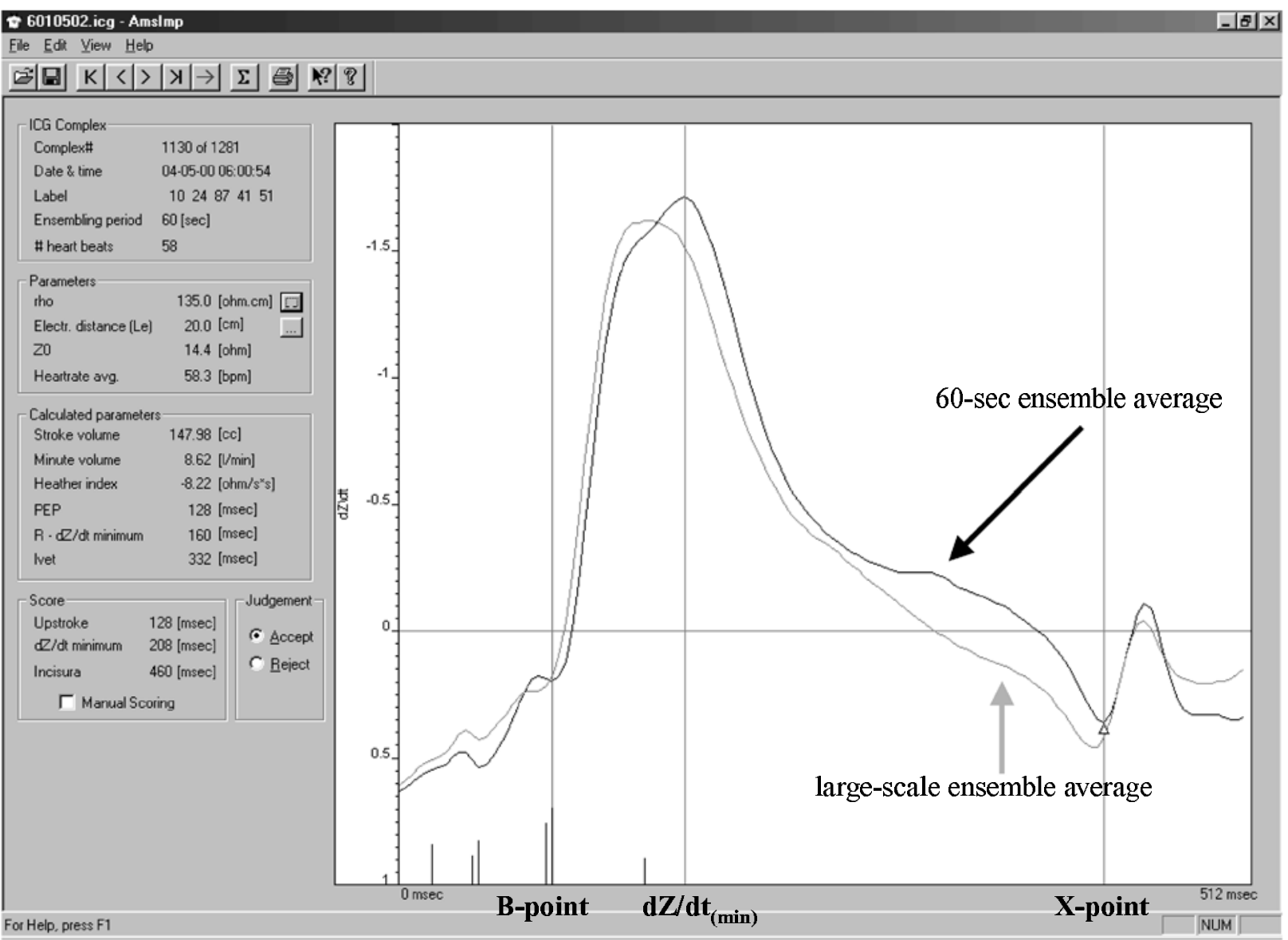

Figure 1B. The same 60-sec ensemble average as in Figure 1A (black), now overlaid with the corresponding large-scale ensemble average (gray).

method $(.05,-.07, .30)$, and for Subjects 10 and 20 for the large-scale ensemble average method $(-.42, .07)$. Visual inspection of the $\mathrm{dZ} / \mathrm{dt}$ waveforms suggested that this could have been due to the ambiguous dZ/dt waveforms of these 4 subjects, particularly with regard to the B-point.

\section{Cross-Method Comparison}

Rather than collapsing across Raters 1 and 2, we analyzed the full set of correlations by way of replication. The mean intraclass correlation coefficients between the cardiac measures obtained with 60-sec and large-scale ensemble averaging was for .80 PEP, .99 for IBI, and .97 for $\mathrm{dZ} / \mathrm{dt}$. Of the coefficients of individual raters, $92.3 \%$ were higher than .80 and $83.3 \%$ were higher than .90 . These high percentages indicate that the relative ranking of the cardiac function measures remained fairly constant regardless the method of ensemble averaging. Again, Subjects 5, 10, 12, and 20, who had the most ambiguous B-points, yielded the lowest cross-method correlation for PEP, although in only one of the raters (.78,
$.96, .96$, and .98 for Rater 1 , and $-.03,-.24, .12$, and .08 for Rater 2, respectively).

\section{Individual Differences in PEP Profiles}

Figure 3 shows the 24-h PEP registrations for 4 subjects selected to illustrate the extremes of the continuum of PEP profiles found in this population. The numbers on the $x$ axis represent consecutive fixed periods over the 24-h ambulatory monitoring period (e.g., as described for Subject 3 in Table 3). Subjects 3 and 9 show a strong wake-sleep reactivity on absolute PEP. In contrast, Subjects 13 and 16 show a relatively constant absolute PEP during the 24-h period. Note that despite the large individual differences in PEP profile, subjects showed similar results with the 60sec ensemble and large-scale ensemble averaging.

\section{Effects of Posture and Activity}

Table 3 provides a description of all cardiac function measures across the three posture/physical load categories: (1) lying during night-time sleep, (2) sitting during light physical load, and (3) medium physical load in 
Table 1

The Various Categories Used for Coding Fixed Periods in 24-h Ambulatory Data

\begin{tabular}{|c|c|}
\hline Category & Codes for Fixed Periods \\
\hline Activity & $\begin{array}{l}\text { Desk work (PC work, administration, reading and } \\
\text { writing), manual labor (construction, repair, machine } \\
\text { operation), housekeeping activities (cooking, ironing), } \\
\text { active recreational (dancing, musical instrument), } \\
\text { active transportation (car or motorcycle driving, } \\
\text { walking, bicycling), public transportation (bus, train, } \\
\text { or car passenger), dyadic conversation at work } \\
\text { (includes telephone), dyadic conversation at home } \\
\text { (includes telephone), passive recreational (TV, read- } \\
\text { ing, attending a play/musical/opera, movie), eating } \\
\text { and drinking, sleep, unknown }\end{array}$ \\
\hline Physical load & $\begin{array}{l}\text { None/sleep, light, moderate, heavy, very heavy, } \\
\text { unknown }\end{array}$ \\
\hline Posture & $\begin{array}{l}\text { Lying, sitting, standing, walking, lying/sitting, sit/ } \\
\text { standing, sit/stand/walking, stand/walking, bicycling, } \\
\text { unknown }\end{array}$ \\
\hline Location & $\begin{array}{l}\text { At home, at work, public space (restaurant, traffic, } \\
\text { hospital), at home of family or friends, outdoors, un- } \\
\text { known }\end{array}$ \\
\hline Social situation & $\begin{array}{l}\text { Alone in private space, with significant others (partner/ } \\
\text { children), with friends, with colleagues, with others } \\
\text { (not significant others, family, or friends), unknown }\end{array}$ \\
\hline
\end{tabular}

all postures except sitting. Large individual differences in absolute PEP were obvious from the relatively large $S D$ across the whole group. Part of the intraindividual variance in PEP was due to changes in posture and physical activity, but as shown in Table 3, high PEP variance was found even within the three posture/physical load categories. For instance, the mean $S D$ across all 60-sec averages obtained when the subjects were sitting was 4.27 (range $=1.49-12.87)$ for Rater 1 and $5.13($ range $=$ 0.93-17.07) for Rater 2. And the mean $S D$ across all available large-scale ensembled averages from sitting periods were 3.73 (range $=0.00-12.95$ ) for Rater 1 and 3.46 (range $=0.00-7.12$ ) for Rater 2 .

A three-level repeated measures MANOVA tested for main and interaction effects of rater, posture/physical load, and method of ensemble averaging. No main or interaction effects involving rater were found. With increasing physical load, IBI $[F(2,19)=128.49, p<.000]$ and PEP $[F(2,19)=19.21, p<.000]$ decreased. Absolute IBI and PEP levels did not differ across the two ensemble averaging methods. For dZ/dt amplitude, a significant interaction between ensemble averaging methods and posture/physical load was found $[F(2,19)=18.84$, $p<.000]$. The $\mathrm{dZ} / \mathrm{dt}$ amplitude increased with increasing physical load, but this increase was smaller in the largescale ensemble average method than in the 60-sec ensemble average method.

\section{DISCUSSION}

This study tested whether large-scale ensemble averaging of ambulatory ICGs across periods with fixed activity, posture, social situation, and location would yield valid estimates of the PEP across these periods. As a val- idating criterion, we used the mean of all, interactively scored, 60-sec ensemble averages across these same periods. Intraclass correlations between measures derived by 60 -sec ensemble averages and measures derived by large-scale ensemble averaging were generally very high. In addition, both types of ensemble averaging methods showed good interrater reliability across random pairings of seven different raters.

In spite of the good correspondence overall, poor cross-method correlation was found for PEP in 4 subjects. This did not appear to be due to the use of largescale ensemble averaging. In these 4 subjects, even within the two ensembling methods separately, much lower interrater reliability was found than in any of the other subjects. Inspection of these subjects showed that both low interrater reliability and low cross-method comparison were due to ambiguous $\mathrm{dZ} / \mathrm{dt}$ waveforms, which made the choice of a stable B-point difficult. Most raters identified the same 4 subjects as having problematic ICG waveforms. A conservative strategy would be to remove all subjects with such ambiguous B-points from further analyses. Clearly, this would cause the loss of "expensive" ambulatory data. Moreover, it remains to be shown whether such subjects are a random selection or whether the occurrence of ambiguous $\mathrm{dZ} / \mathrm{dt}$ waveforms could somehow be confounded with age or cardiovascular health. A more valid future strategy, therefore, would be the use of an additional criterion for B-point detection, possibly using the phonocardiogram.

The present study adds to the evidence of the feasibility of PEP recording in a naturalistic, ambulatory setting (de Geus \& van Doornen, 1996; Willemsen et al., 1996). Intriguingly, large individual differences in absolute PEP were found, as well as differences in within-subjects PEP changes across the day (i.e., as reflected in changes from day to night and in the variation in $S D$ s). This replicates the $S D$ s reported over a 48 -h period for 2 subjects (PEP $S D$ s were 5.0 and 9.6, respectively) by Miller and $\mathrm{He}-$ lander (1979) in a very early attempt at ambulatory ICG recording using band electrodes. In laboratory studies on individual differences, the focus has been on PEP reactivity, or within-subjects decreases in PEP from a baseline condition in response to stressors. PEP reactivity has proven to be a reliable indicator of changes in cardiac sympathetic drive in studies manipulating $\beta$-adrenergic drive by epinephrine infusion (Mezzacappa, Kelsey, \& Katkin, 1999; Svedenhag, Martinsson, Ekblom, \& Hjemdahl, 1986), adrenoceptor blockade (Cacioppo et al., 1994; W. S. Harris, Schoenfeld, \& Weissler, 1967), exercise (Krzeminski et al., 2000; Miyamoto et al., 1983; Smith et al., 1989), or emotional stress (Berntson et al., 1994; Newlin \& Levenson, 1979; Sherwood et al., 1986). Test-retest studies of PEP reactivity to a variety of manipulations have shown it to be a stable individual characteristic (Nelesen, Shaw, Ziegler, \& Dimsdale, 1999; Sherwood \& Turner, 1993), with generalizability coefficients over tasks and sessions of .57 and .68 in two independent studies (Kamarck et al., 1992). In addition, PEP 
Table 2

A Representative Example of the Coding of Fixed Periods of a 24-h Registration

\begin{tabular}{|c|c|c|c|c|c|c|}
\hline $\mathrm{N}$ & Start time & Activity & Physical Load & Posture & Location & Social Situation \\
\hline 1 & $10: 20: 37$ & Dyadic conversation at home & Light & Sit/stand & Home & With others \\
\hline 2 & $10: 32: 43$ & Active transportation & Moderate & Sit/stand/walking & Public space & With children \\
\hline 3 & $10: 52: 35$ & Dyadic conversation at home & Light & Sitting & At friends" home & With children \\
\hline 4 & $11: 45: 21$ & Active transportation & Moderate & Sit/stand/walking & Public space & With children \\
\hline 5 & $12: 02: 56$ & Housekeeping & Moderate & Sit/stand/walking & At home & With children \\
\hline 6 & 12:31:08 & Active transportation & Heavy & Bicycling & Outdoors & With children \\
\hline 7 & $12: 42: 52$ & Manual labor & Light & Stand/walking & Outdoors & With others \\
\hline 8 & $12: 52: 31$ & Active transportation & Moderate & Bicycling & Outdoors & Alone \\
\hline 9 & $12: 57: 35$ & Manual labor & Moderate & Sit/stand/walking & Home & With children \\
\hline 10 & 13:30:47 & Passive recreational & Light & Sitting & Home & Alone \\
\hline 11 & $13: 51: 53$ & Manual labor & Moderate & Sit/stand/walking & Home & Alone \\
\hline 12 & 14:07:36 & Passive recreational & Light & Sitting & Home & Alone \\
\hline 13 & $14: 43: 59$ & Manual labor & Moderate & Sit/stand/walking & Home & With children \\
\hline 14 & $15: 14: 34$ & Active transportation & Moderate & Bicycling & Outdoors & Alone \\
\hline 15 & $15: 25: 42$ & Active transportation & Heavy & Bicycling & Outdoors & With children \\
\hline 16 & $15: 35: 45$ & Manual labor & Light & Sit/stand/walking & At home & With children \\
\hline 17 & $15: 59: 54$ & Active recreational & Moderate & Sit/stand/walking & At home & With children \\
\hline 18 & $16: 41: 52$ & Manual labor & Light & Sit/stand/walking & At home & With children \\
\hline 19 & 17:04:11 & Eating and drinking & Light & Sitting & At home & With children \\
\hline 20 & $17: 43: 37$ & Manual labor & Moderate & Sit/stand/walking & At home & With children \\
\hline 21 & 18:01:54 & Active recreational & Light & Sitting & At home & With children \\
\hline 22 & $18: 42: 36$ & Passive recreational & Light & Sitting & At home & With children \\
\hline 23 & 19:00:47 & Manual labor & Moderate & Sit/stand/walking & At home & With children \\
\hline 24 & 19:31:13 & Manual labor & Moderate & Sit/stand/walking & At home & Unknown \\
\hline 25 & 20:02:06 & Passive recreational & Light & Sitting & At home & Unknown \\
\hline 26 & 20:16:09 & Manual labor & Light & Sit/stand/walking & At home & With colleagues \\
\hline 27 & $20: 40: 17$ & Dyadic conversation at work & Light & Sitting & At home & With colleagues \\
\hline 28 & $21: 38: 51$ & Passive recreational & Light & Sitting & At home & With partner \\
\hline 29 & $22: 38: 51$ & Passive recreational & Light & Sitting & At home & With partner \\
\hline 30 & $23: 02: 14$ & Manual labor & Light & Sit/stand/walking & At home & With partner \\
\hline 31 & $23: 15: 27$ & First hour sleep & Sleep & Lying & At home & With partner \\
\hline 32 & $0: 15: 35$ & Second hour sleep & Sleep & Lying & At home & With partner \\
\hline 33 & $1: 15: 39$ & Third hour sleep & Sleep & Lying & At home & With partner \\
\hline 34 & $2: 15: 48$ & Fourth hour sleep & Sleep & Lying & At home & With partner \\
\hline 35 & $3: 15: 39$ & Fifth hour sleep & Sleep & Lying & At home & With partner \\
\hline 36 & $4: 15: 35$ & Sixth hour sleep & Sleep & Lying & At home & With partner \\
\hline 37 & $5: 15: 29$ & Seventh hour sleep & Sleep & Lying & At home & With partner \\
\hline 38 & $6: 15: 51$ & Eighth hour sleep & Sleep & Lying & At home & With partner \\
\hline 39 & $7: 15: 59$ & Ninth hour sleep & Sleep & Lying & At home & With partner \\
\hline 40 & 7:34:57 & Manual labor & Light & Sit/stand/walking & At home & With children \\
\hline 41 & 7:56:06 & Manual labor & Moderate & Sit/stand/walking & At home & With children \\
\hline
\end{tabular}

Note-The start time, type of current activity, physical load, posture, location, and social situation are given for each fixed period. For instance, the first fixed period started at 10:20:37 hours when the researcher visited the subject at home for fitting the ambulatory devices and receiving instructions. Note that none of the fixed periods is shorter than $5 \mathrm{~min}$ or longer than $60 \mathrm{~min}$ - that is, nighttime sleep is divided into multiple periods. The subject was a healthy 31 -year-old woman with a body mass index of 18.6.

reactivity systematically correlates to sympathetic effects on hormonal (A. S. Harris et al., 2001; McCubbin, Richardson, Langer, Kizer, \& Obrist, 1983), cardiovascular (Neumann \& Waldstein, 2001), and immune (Cacioppo et al., 1995) parameters.

Much less is known about the significance of interindividual differences in absolute PEP level. In an encouraging study by Cacioppo et al. (1994), absolute PEP levels closely reflected pharmacologically derived sympathetic drive. In addition, preliminary evidence from studies in twins in our lab shows that monozygotic twin resemblance for PEP is very high, suggesting absolute PEP to be a stable, heritable trait. Taken together, these findings suggest that ambulatory PEP levels might be used as an index of ambulatory sympathetic drive, a mea- sure previously available only by catecholamine sampling, with a number of methodological problems (Esler et al., 1990; Hjemdahl, 1987). Caution is in order, however. First of all, functional heart responses (like PEP) do not just reflect sympathetic drive (i.e., the amount of firing of the postganglionic noradrenergic neurons in the sympathetic chain ganglia). They also depend on the efficiency of neurotransmission in the ventricular adrenergic synapses, which can vary as a function of cardiac $\beta$-receptor density or affinity. In humans, effects of noradrenergic neurotransmission on ventricular contractility depends mainly on $\beta 1$-receptors, but functional contribution of $\beta 2$-receptors in response to circulating adrenaline from adrenal medullarly secretion is likely to contribute as well. 


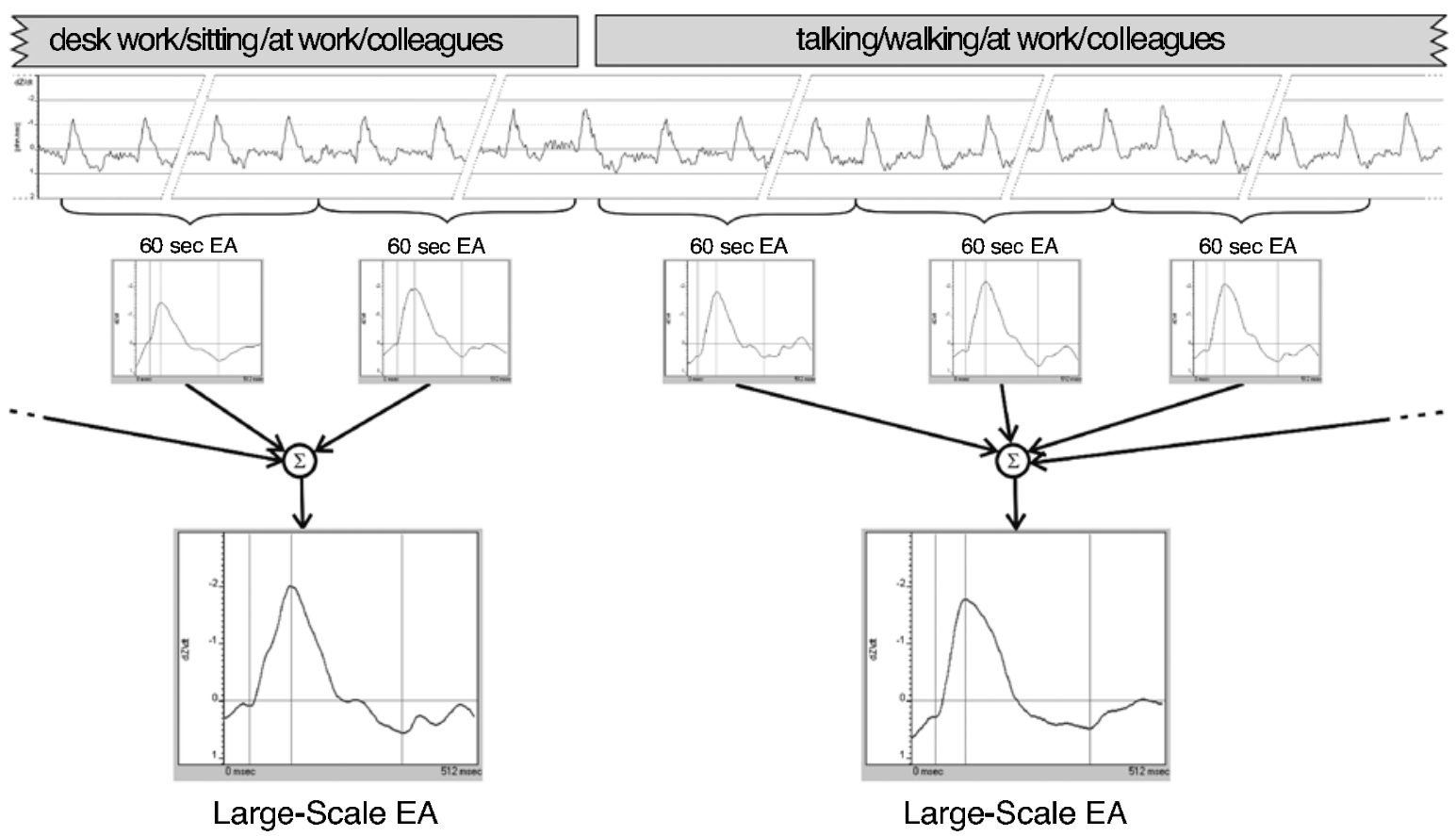

Figure 2. Schematic representation of the ensemble averaging (EA) of single-beat impedance waveforms across 60 -sec periods (upper part) and the large-scale ensemble averaging of these 60 -sec ensemble averaged waveforms across periods with fixed posture, activity, location, and social situation (lower part).
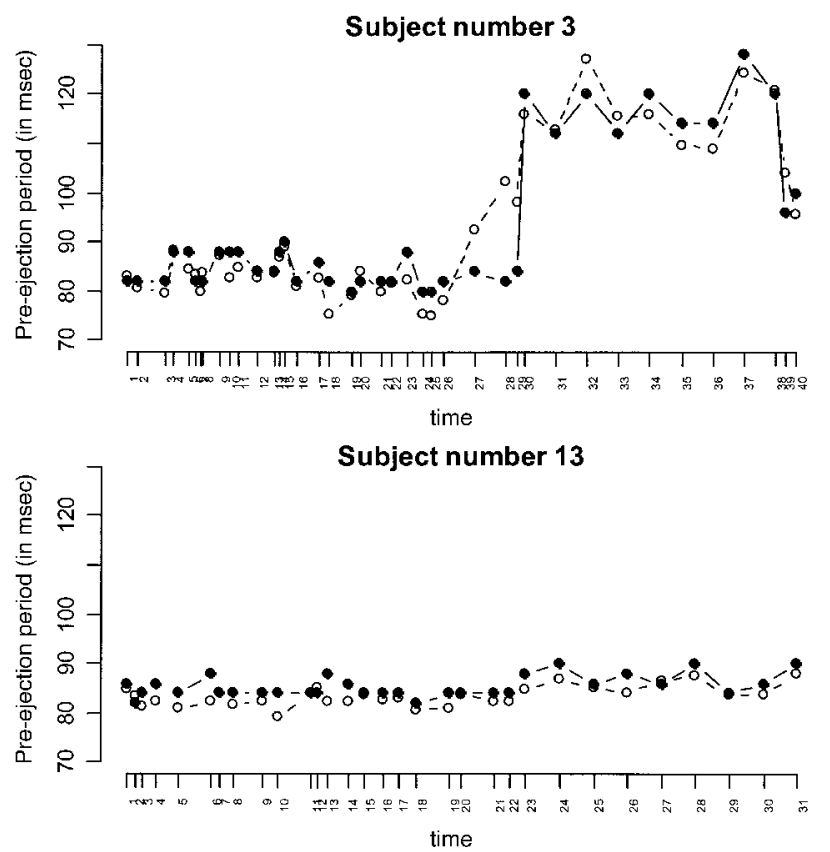

Subject number 9
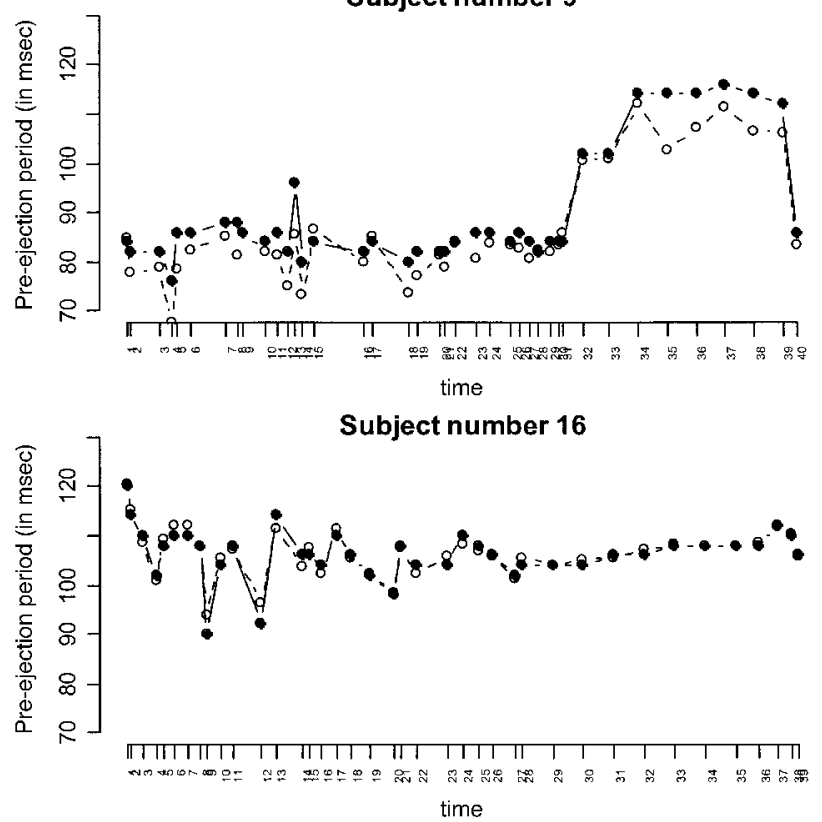

Figure 3. Twenty-four-hour profiles of pre-ejection period for 4 representative subjects. Lines represent the mean scores of the two raters. The dashed lines represent the mean PEP from the 60-sec ensemble averages across periods with fixed posture, activity, location, and social situation. The solid lines represent the large-scale ensemble averaged PEP over these same fixed periods. On the $x$ axis, the start of each successive fixed time period is indicated as a tick-mark. The irregularity in the distribution of the tick-marks on the $x$-axis is due to the differences in duration of the fixed time periods (see text for further explanation). 
Table 3

Means and Standard Deviations of PEP, $\mathbf{d Z / d t} \mathrm{t}_{(\mathrm{min})}$, and IBI Across Combined Posture/Physical Load Categories

\begin{tabular}{|c|c|c|c|c|c|c|c|c|}
\hline \multirow[b]{3}{*}{ Variable/Category } & \multicolumn{4}{|c|}{ 60-sec Ensemble Averages } & \multicolumn{4}{|c|}{ Large-Scale Ensemble Averages } \\
\hline & \multicolumn{2}{|c|}{ Rater 1} & \multicolumn{2}{|c|}{ Rater 2} & \multicolumn{2}{|c|}{ Rater 1} & \multicolumn{2}{|c|}{ Rater 2} \\
\hline & $M$ & $S D$ & $M$ & $S D$ & $M$ & $S D$ & $M$ & $S D$ \\
\hline \multicolumn{9}{|l|}{ PEP (in msec) } \\
\hline Nighttime sleep/lying & 102.57 & 15.61 & 103.93 & 17.89 & 102.90 & 15.83 & 101.37 & 20.53 \\
\hline Light physical load/sitting & 97.15 & 18.96 & 96.15 & 19.52 & 98.12 & 19.05 & 96.33 & 19.53 \\
\hline Moderate physical load/not lying or sitting & 94.64 & 16.97 & 93.36 & 17.73 & 95.31 & 16.75 & 93.93 & 17.10 \\
\hline \multicolumn{9}{|l|}{$\mathrm{dZ} / \mathrm{dt}_{(\min )}($ in $\Omega / \mathrm{sec})$} \\
\hline Nighttime sleep/lying & -1.042 & .257 & -1.038 & .241 & -1.007 & .246 & -1.006 & .237 \\
\hline Light physical load/sitting & -1.250 & .292 & -1.249 & .293 & -1.199 & .283 & -1.199 & .278 \\
\hline Moderate physical load/not lying or sitting & -1.349 & .312 & -1.351 & .309 & -1.261 & .295 & -1.260 & .293 \\
\hline \multicolumn{9}{|l|}{ IBI (in msec) } \\
\hline Nighttime sleep/lying & $1,006.98$ & 156.92 & $1,006.75$ & 157.67 & $1,005.98$ & 156.33 & $1,005.68$ & 157.07 \\
\hline Light physical load/sitting & 790.84 & 141.19 & 790.64 & 140.89 & 791.49 & 141.10 & 791.15 & 140.91 \\
\hline Moderate physical load/not lying or sitting & 705.93 & 97.39 & 706.98 & 102.01 & 706.64 & 101.62 & 706.12 & 101.06 \\
\hline
\end{tabular}

Second, (pathological) cardiac dimensions can severely distort the relationship between sympathetic drive and absolute PEP. Prolonged PEP has been found in patients with aortic stenosis (Moene, Mook, Kruizinga, Bergstra, \& Bossina, 1975) and in patients with primary pulmonary hypertension drive (Shigematsu, Hamada, \& Kokubu, 1988). Clearly, this cannot be attributed to low cardiac sympathetic drive, but instead seems to reflect increases in aortic impedance and systemic vascular resistance inducing increased afterload effects. Even in healthy subjects, individual differences in afterload may distort the relation of PEP with sympathetic drive to the heart. Independent of contractility, PEP will become longer when the end-diastolic aortic pressure, or afterload, increases. This simply derives from the fact that a larger intraventricular pressure buildup is needed before the aortic valves are pushed open.

Third, PEP also strongly depends on venous return and the degree of myocardial muscle stretch induced (preload). Cardiac contraction force increases with increased end-diastolic myocardial muscle stretch - the normal length-tension relationship in any sarcomere. This preload effect on cardiac contraction ensures that systemic and pulmonary circulation remain matched (Frank Starling mechanism). Independent of contractility, therefore, PEP shortens with increasing ventricular preload. In an ambulatory setting, the concerns raised about the confounding effects of changes in preload and afterload apply in full. Venous return decreases more during standing up than during sitting, and increases during lying down (sleep). The effects of physical activity on preload depend very much on type of activity; static contraction will reduce blood flow, whereas dynamic muscular action will enhance venous return.

In short, measuring individual differences in sympathetic drive by using absolute PEP confounds sympathetic drive with individual differences in adrenoceptor functioning as well as with individual differences in preload and afterload. Thus it seems sensible to use ambulatory PEP mainly in a within-subjects design. Withinsubjects changes in PEP across the day can easily be recast in terms of various reactivity measures (e.g., sleep vs. wake, standard deviation, or reactivity to specific events). These reactivity measures, in turn, can be used in a between-subjects comparison-for instance, to see whether the sleep-daytime differences are larger in anxious depressed subjects than in controls. To obtain such measures, at least for unambiguous ambulatory ICGs, large-scale ensemble averaging was shown to validly recapture the information in the original 60-sec ensemble averages while substantially reducing the total amount of visual inspection needed. Thus large-scale ensemble averaging makes the application of the recently developed ambulatory ICG monitors (Cybulski, 2000; Nakonezny et al., 2001; Sherwood et al., 1998; Willemsen et al., 1996) feasible even for large-scale epidemiological studies.

\section{REFERENCES}

Alfie, J., Waisman, G. D., Galarza, C. R., Magi, M. I., Vasvari, F., Mayorga, L. M., \& CAmera, M. I. (1995). Relationship between systemic hemodynamics and ambulatory blood pressure level [sic] are sex dependent. Hypertension, 26, 1195-1199.

Berntson, G. G., Cacioppo, J. T., Binkley, P. F., Uchino, B. N. Quigley, K. S., \& Fieldstone, A. (1994). Autonomic cardiac control. III. Psychological stress and cardiac response in autonomic space as revealed by pharmacological blockades. Psychophysiology, 31, 599-608.

Boomsma, D. I., Beem, A. L., van den Berg, M., Dolan, C. V., Koopmans, J. R., Vink, J. M., de Geus, E. J. C., \& Slagboom, P. E. (2000). Netherlands twin-family study of anxious depression (NETSAD). Twin Research, 3, 323-334.

Boomsma, D. I., de VRies, J., \& ORLebeke, J. F. (1989). Methodology: Comparison of spot and band impedance cardiogram electrodes across different tasks. Psychophysiology, 26, 695-699.

Bosch, J. A., de Geus, E. J. C., Kelder, A., Veerman, E. C., HoogStraten, J., \& Amerongen, A. V. (2001). Differential effects of active versus passive coping on secretory immunity. Psychophysiology, 38, 836-846.

Bosch, J. A., de Geus, E. J. C., Ligtenberg, T. J., Nazmi, K., Veerman, E. C., Hoogstraten, J., \& Amerongen, A. V. (2000). Salivary MUC5B-mediated adherence (ex vivo) of Helicobacter pylori during acute stress. Psychosomatic Medicine, 62, 40-49.

Burgess, H. J., Kleiman, J., \& Trinder, J. (1999). Cardiac activity during sleep onset. Psychophysiology, 36, 298-306.

Burgess, H. J., Sletten, T., SAvic, N., Gilbert, S. S., \& Dawson, D. 
(2001). Effects of bright light and melatonin on sleep propensity, temperature, and cardiac activity at night. Journal of Applied Physiology, 91, 1214-1222.

Burgess, H. J., Trinder, J., \& Kim, Y. (1999). Cardiac autonomic nervous system activity during presleep wakefulness and stage 2 NREM sleep. Journal of Sleep Research, 8, 113-122.

Burgess, H. J., Trinder, J., Kim, Y., \& LuKe, D. (1997). Sleep and circadian influences on cardiac autonomic nervous system activity. American Journal of Physiology, 273(4 Pt. 2), H1761-H1768.

Cacioppo, J. T., Berntson, G. G., Binkley, P. F., Quigley, K. S., Uchino, B. N., \& Fieldstone, A. (1994). Autonomic cardiac control. II. Noninvasive indices and basal response as revealed by autonomic blockades. Psychophysiology, 31, 586-598.

Cacioppo, J. T., Malarkey, W. B., Kiecolt-Glaser, J. K., Uchino, B. N., Sgoutas-Emch, S. A., Sheridan, J. F., Berntson, G. G., \& GLASER, R. (1995). Heterogeneity in neuroendocrine and immune responses to brief psychological stressors as a function of autonomic cardiac activation. Psychosomatic Medicine, 57, 154-164.

Cy вulski, G. (2000). Letter to the editor. Journal of Applied Physiology, 88, 1509-1510.

Debski, T. T., Zhang, Y., Jennings, J. R., \& Kamarck, T. W. (1993). Stability of cardiac impedance measures: Aortic opening (B-point) detection and scoring. Biological Psychology, 36, 63-74.

DE Geus, E. J. C., \& van Doornen, L. J. P. (1996). Ambulatory assessment of parasympathetic/sympathetic balance by impedance cardiography. In J. Fahrenberg \& M. Myrtek (Eds.), Ambulatory assessment: Computer-assisted psychological and psychophysiological methods in monitoring and field studies (pp. 141-163). Göttingen, Germany: Hogrefe \& Huber.

de Geus, E. J. C., Willemsen, G. H. M., Klaver, C. H. A. M., \& van Doornen, L. J. P. (1995). Ambulatory measurement of respiratory sinus arrhythmia and respiration rate. Biological Psychology, 41, 205-227.

Esler, M., Jennings, G., Lamber, G., Meredith, I., Horne, M., \& EISENHOFER, G. (1990). Overflow of catecholamine neurotransmitters to the circulation: Source, fate and functions. Physiological Review, 70, 963-985.

Groza, P., Vrancianu, R., Lazar, M., Baevski, R. M., \& Funtova, V. L. (1984). Systolic time intervals after a seven-day orbital flight. Advances in Space Research, 4, 11-14.

Harris, A. S., Burgess, H. J., \& Dawson, D. (2001). The effects of day-time exogenous melatonin administration on cardiac autonomic activity. Journal of Pineal Research, 31, 199-205.

Harris, W. S., Schoenfeld, C. D., \& Weissler, A. M. (1967). Effects of adrenergic receptor activation and blockade on the systolic preejection period, heart rate, and arterial pressure in man. Journal of Clinical Investigation, 46, 1704-1714.

HJEMdAHL P. (1987). Physiological aspects on catecholamine sampling. Life Sciences, 41, 841-844.

Holmes, A. L., Burgess, H. J., \& Dawson, D. (2002). Effects of sleep pressure on endogenous cardiac autonomic activity and body temperature. Journal of Applied Physiology, 92, 2578-2584.

Houtveen, J., Rietveld, S., \& De Geus, E. J. C. (2002). Contribution of tonic vagal modulation of heart rate, central respiratory drive, respiratory depth, and respiratory frequency to respiratory sinus arrhythmia during mental stress and physical exercise. Psychophysiology, 39, 427-436.

Kamarck, T. W., Jennings, J. R., Debski, T. T., Glickman-Weiss, E. Johnson, P. S., EDDY, M. J., \& MANucK, S. B. (1992). Reliable measures of behaviorally-evoked cardiovascular reactivity from a PCbased test battery: Results from student and community samples. Psychophysiology, 29, 17-28.

Kelsey, R. M., \& Guethlein, W. (1990). An evaluation of the ensemble averaged impedance cardiogram. Psychophysiology, 27, 24-33.

Kelsey, R. M., Reiff, S., Wiens, S., Schneider, T. R, Mezzacappa, E. S., \& Guethlein, W. (1998). The ensemble-averaged impedance cardiogram: An evaluation of scoring methods and interrater reliability. Psychophysiology, 35, 337-340.

Kizakevich,P. N., McCartney, M. L., Hazucha, M. J., Sleet, L. H., Jochem, W. J., Hackney, A. C., \& Bolick, K. (2000). Noninvasive ambulatory assessment of cardiac function in healthy men exposed to carbon monoxide during upper and lower body exercise. European Journal of Applied Physiology, 83, 7-16.

Krzeminski, K., Kruk, B., Nazar, K., Ziemba, A. W., Cybulski, G., \& NIEWIADOMSKI, W. (2000). Cardiovascular, metabolic and plasma catecholamine responses to passive and active exercises. Journal of Physiological Pharmacology, 51, 267-278.

Light, K. C., Kothandapani, R. V., \& Allen, M. T. (1998). Enhanced cardiovascular and catecholamine responses in women with depressive symptoms. International Journal of Psychophysiology, 28, 157166

Matthews, K. A., Flory, J. D., Owens, J. F., Harris, K. F., \& Berga, S. L. (2001). Influence of estrogen replacement therapy on cardiovascular responses to stress of healthy postmenopausal women. Psychophysiology, 38, 391-398.

McCubbin, J. A., Richardson, J. E., Langer, A. W., Kizer, J. S., \& OBRist, P. A. (1983). Sympathetic neuronal function and left ventricular performance during behavioral stress in humans: The relationship between plasma catecholamines and systolic time intervals. Psychophysiology, 20, 102-110.

Mezzacappa, E. S., Kelsey, R. M., \& Katkin, E. S. (1999). The effects of epinephrine administration on impedance cardiographic measures of cardiovascular function. International Journal of Psychophysiology, 31, 189-196.

Miller, J. C., \& Helander, M. (1979). The 24 hour cycle and nocturnal depression of human cardiac output. Aviation, Space, \& Environmental Medicine, 50, 1139-1144.

Miyamoto, Y., Higuchi, J., Abe, Y., Hiura, T., Nakazono, Y., \& MiKami, T. (1983). Dynamics of cardiac output and systolic time intervals in supine and upright exercise. Journal of Applied Physiology, 55, 1674-1681.

Moene, R. J., Mook, G. A., Kruizinga, K., Bergstra, A., \& Bossina, K. K. (1975). Value of systolic time intervals in assessing severity of congenital aortic stenosis in children. British Heart Journal, 37, 1113-1122.

Muzi, M., Ebert, T. J., Tristani, F. E., Jeutter, D. C., Barney, J. A., \& SмIтн, J. J. (1985). Determination of cardiac output using ensembleaveraged impedance cardiograms. Journal of Applied Physiology, 58, 200-205.

Nakonezny, P. A., Kowalewski, R. B., ERnst, J. M., Hawkley, L. C., Lozano, D. L., Litvack, D. A., Berntson, G. G., Sollers, J. J., Kizakevich. P., CACiopPo, J. T., \& Lovallo, W. R. (2001). New ambulatory impedance cardiograph validated against the Minnesota Impedance Cardiograph. Psychophysiology, 38, 465-473.

Nelesen, R. A., Shaw, R, Ziegler, M. G., \& Dimsdale, J. E. (1999) Impedance cardiography-derived hemodynamic responses during baroreceptor testing with amyl nitrite and phenylephrine: A validity and reliability study. Psychophysiology, 36, 105-108.

Neumann, S. A., \& Waldstein, S. R. (2001). Similar patterns of cardiovascular response during emotional activation as a function of affective valence and arousal and gender. Journal of Psychosomatic Research, 50, 245-253.

Newlin, D. B., \& Levenson, R. W. (1979). Pre-ejection period: Measuring beta-adrenergic influences upon the heart. Psychophysiology, 16, 546-553.

Ring, C., Carroll, D., Willemsen, G., Cooke, J., Ferraro, A., \& DrAYSON, M. (1999). Secretory immunoglobulin A and cardiovascular activity during mental arithmetic and paced breathing. Psychophysiology, 36, 602-609.

Sherwood, A., Allen, M. T., Fahrenberg, J., Kelsey, R. M., Lovallo, W. R., \& van Doornen, L. J. P. (1990). Committee report: Methodological guidelines for impedance cardiography. Psychophysiology, 27, 1-23.

Sherwood, A., Allen, M. T., Obrist, P. A., \& Langer, A. W. (1986) Evaluation of beta-adrenergic influences on cardiovascular and metabolic adjustments to physical and psychological stress Psychophysiology, 23, 89-104.

Sherwood, A., McFetridge, J., \& Hutcheson, J. S. (1998). Ambulatory impedance cardiography: A feasibility study. Journal of Applied Physiology, 85, 2365-2369. 
Sherwood, A., \& Turner, J. R. (1993). Postural stability of hemodynamic responses during mental challenge. Psychophysiology, 30, 237-244.

Shigematsu, Y., Hamada, M., \& KoKubu, T. J. (1988). Significance of systolic time intervals in predicting prognosis of primary pulmonary hypertension. Cardiology, 18, 1109-1114.

Siegel, J. H, \& Sonnenblick, E. H. (1963). Isometric time-tension relations as an index of myocardial contractility. Circulation Research, $12,597$.

Smith, J. J., Muzi, M., Barney, J. A., Ceschi, J., Hayes, J., \& Ebert, T. J. (1989). Impedance-derived cardiac indices in supine and upright exercise. Annals of Biomedical Engineering, 17, 507-515.

Staessen, J. A., Asmar, R, de Buy zere, M., Imai, Y., Parati, G., Shimada, K., Stergiou, G., Redon, J., Verdecchia, P., \& ParticiPants of The 200 i Consensus Conference on Ambulatory Blood Pressure Monitoring (2001). Task Force II: Blood pressure measurement and cardiovascular outcome. Blood Pressure Monitor, 6, 355-370.

Svedenhag,J., Martinsson, A., Ek blom, B., \& Hiemdahl, P. (1986).
Altered cardiovascular responsiveness to adrenaline in endurancetrained subjects. Acta Physiologica Scandinavia, 126, 539-550.

VERDECCHIA,P. (2000). Prognostic value of ambulatory blood pressure: Current evidence and clinical implications. Hypertension, 35, 844. VRiJkotte, T. G. M., Riese, H., \& DE Geus, E. J. C. (2001). Effects of workstress on ambulatory heart rate, heart rate variability, and blood pressure. In J. Fahrenberg \& M. Myrtek (Eds.), Progress in ambulatory assessment (pp. 345-360). Seattle: Hogrefe \& Huber

Vrijkotte, T. G. M., van Doornen, L. J. P., \& de Geus, E. J. C. (2000). Effects of workstress on ambulatory blood pressure, heart rate, and heart rate variability. Hypertension, 35, 880-886.

Willemsen, G. H. M., de Geus, E. J. C., Klaver, C. H. A. M., van Doornen, L. J. P., \& Carroll, D. (1996). Methodology: Ambulatory monitoring of the impedance cardiogram. Psychophysiology, 33, 184-193.

(Manuscript received October 22, 2002; revision accepted for publication May 14, 2003.) 